\title{
Safety dose and prediction of immune-related adverse event of nivolumab combined with IFN- $\beta$ eta in patients with advanced melanoma
}

OTaku Fujimura'1), Yumi Kambayashi'1), Sadanori Furudate1), Takanori Hidaka1), Hisayuki Tono'), Yota Sato'), Kayo Tanita ${ }^{1)}$, Akira Hashimoto'), Setsuya Aiba ${ }^{1)}$

1) Department of Dermatology, Tohoku University Graduate School of Medicine, Sendai, Japan

Background

Material and Methods

Nivolumab for advanced melanoma

Nivolumab significantly prolongs survival in patients with metastatic melanoma, but only $31 \sim 43 \%$ of patients who receive nivolumab monotherapy experience objective tumor regression [1,2].

Therefore, reagents that enhance the antitumor immune response induced by nivolumab are necessary to further optimize its use for the treatment of advanced melanoma. Although recent studies suggest that such nivolumab-based combined therapy might prove effective for treatment of advanced melanoma [1], the associated risk of immune-related adverse events (irAE), such as severe hepatitis, interstitial pneumonia, colitis, type 1 diabetes mellitus, hypophysitis, or myasthenia gravis, is an important consideration

\section{Interferon beta (IFN- $\boldsymbol{\beta}$ )}

IFN- $\beta$ inhibits the growth of melanoma cells and induces crosspriming of CD8+ T cells by dendritic cells in vitro and in vivo, and has been clinically used for the treatment of malignant melanoma [3].

In melanoma patients, peritumoral injection of IFN- $\beta$ recruits effector cells including CD8+ and TIA1-positive cytotoxic T cells (CTLs) into the tumor microenvironment [4], which might explain one of the treatment of melanoma.

Therapeutic effects of IFN- $\beta$ in combination with anti-PD-1 Abs for B16F10 melanomas.

IFN- $\beta$ enhances the anti-tumor immune response of anti-PD-1 antibodies against B16F10 melanoma by the reduction of CCL22 from Tumor-associated macropha
recruitment of Tregs in vivo [4].
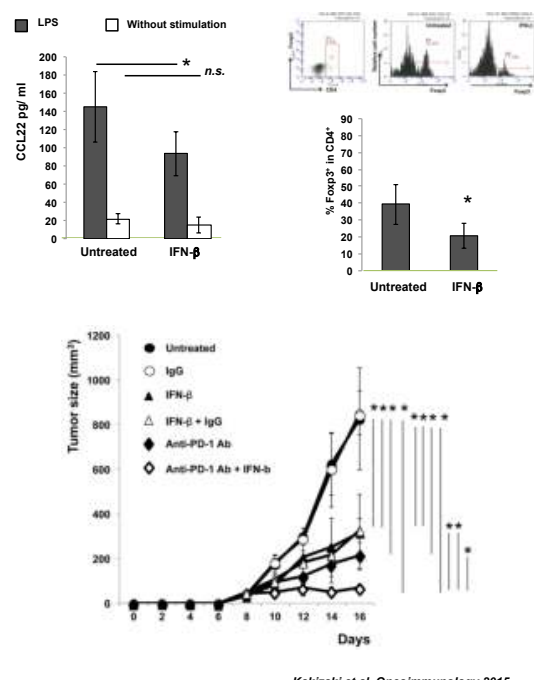

Hypothesis

Since IFN- $\beta$ increases the tumor-infiltrating lymphocytes (TILs) in melanoma, and the number of TILs correlates with the efficacy of nivolumab in various cancers, we hypothesized that IFN- $\beta$ could enhances the therapeutic effects of nivolumab in patents with advanced melanoma.

\section{Purpose}

To explore the potential of this property of IFN- $\beta$ as part of a combination therapy for the treatment of metastatic melanoma patients, first we explored the safety dose of IFN- $\beta$ in combination with nivolumab by a phase 1 trial, using a traditional rule-based $3+$ 3 design, on patients with advanced melanoma.
Patients and methods

Patients were eligible if they had unresectable stage III melanoma, if their tumor was resectable but they had declined resection, or if they had stage IV (patients were staged according to the AJCC Staging Manual, $7^{\text {th }}$ Edition, 2011). Other inclusion criteria were: age of at least 20 years: Eastern Cooperative Oncology Group (ECOG) performance status of 0 to 1 ; and adequate bone marrow and liver function. Exclusion criteria were: active autoimmune disease history of hypersensitivity to nivolumab or IFN- $\beta$; interstitial pneumonia; cance originating in other organs; psychological disorders; and concurrent therapy with any other anti-melanoma chemotherapeutic drugs.

Study design and treatment

This phase 1 trial (UMIN000020222) was an open label, non-randomized,

traditional rule-based $3+3$ design. The intravenous administration of nivolumab was fixed at $2 \mathrm{mg} / \mathrm{kg}$ every 3 weeks (Fig. 1). IFN- $\beta$ was intra-dermally administered at 1 million units, 2 million units, and 3 million units to the three patients in each group at the site of the primary tumor. We set the maximum tolerated dose at the conventional IFN-b therapeutic dose approved in Japan (3 million units).

Assessment

All study patients were evaluated for DLT during the first 6 weeks of treatment. Adverse events were graded using NCI CTCAE version 4.03. DLT were defined goss $(2 \mathrm{mg} / \mathrm{kg}$ ) and IFN- $\beta$ (1 million units) at day 1 (dose design. With 0 of 3 patients exceeding a DIT of grade 3 , three more patients were evaluated at dose level $2+$ of nivolumab $(2 \mathrm{mg} / \mathrm{kg}$ ) and IFN-b $(2$ millio units) at day 1 . With no DLT reported in any of the three patients in level 2 , three more patients were evaluated at dose level $3+$ of nivolumab $(2 \mathrm{mg} / \mathrm{kg}$ and IFN-b (3 million units) at day 1 . With no DLT reported at level 3+, we finalized the study. After the treatment period, patients were assessed every 3 weeks with physical examination, conventional blood examination, and chest radiography, and assessed every 3 months with follow-up computed tomography (CT) scans. The tumor response was clinically evaluated by measuring the longest diameter of the target lesions over time. A partial response (irPR) was defined as a decrease of $>30 \%$, while progressive disease (irPD) was defined as an increase of $>20 \%$, as compared to the baseline measurement. Complete response (irCR) corresponded to the disappearance of all target lesions. We measured tumors 3 months and 6 months after the treatment period

\section{Study oversight}

The study protocol and all amendments were approved by the institutional revew board at Tohoku University Graduate School of Medicine (2016-2-023). The study was conducted in accordance with the Declaration of Helsinki and Confered clinical $\mathrm{H}$. Conference on Harmonisation. All patients provided winten informed consent provide ovesicht. registered with UMIN (UMINO00020222).

An open label, non-randomized, traditional rule-based $3+3$ design

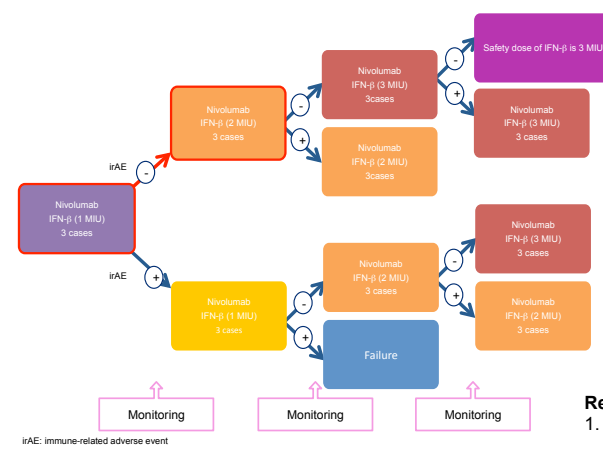

Conclusion

Of the nine patients who received this combined therapy, none experienced dose-limiting toxicities, and all completed the treatmen phase of the study, suggesting that the safe dose of IFN- $\beta$, when combined with nivolumab, is 3 million units.

To determine the efficacy of this combination therapy, further phase I trials are required.

Immune-related adverse events (irAE) might be predicted by serum levels of SCD163 and CXCL5 at day 42.
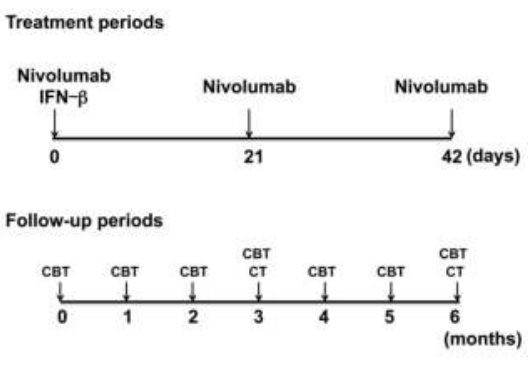

$\underline{\text { Results }}$

Table 1
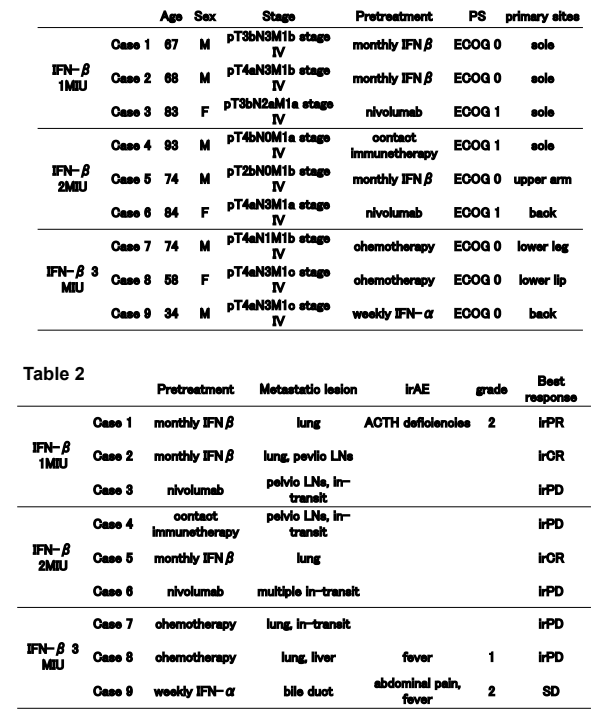

Fig. 1: Change levels of serum SCD163 and CXCL5 at day 42.

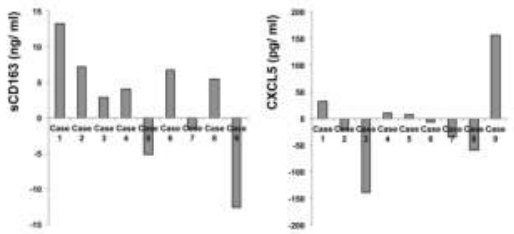

The serum levels of SCD163 (A) and CXCL5 (B) from each patient at day 0 and day 42 were examined by ELISA. These data represent changes of serum SCD163 and CXC15 at day 42 compedat

eferences

Combined Nivolumab and Ipilimumab or Monotherapy in Untreated Melanoma. N Engl J Med. 2015;373:23-34.

2. Topalian SL et al. Survival, durable tumor remission, and long-term safety in patients with advanced melanoma receiving nivolumab. $\mathrm{J}$ Clin Oncol. 2014;32:1020-1030.

3. Aoyagi $\mathrm{S}$, et al. Sequential local injection of low-dose interferon-beta for maintenance therapy in stage II and III melanoma: a single-institution matched case-control study. Oncology. 2012;82:139-146.

Fujimura T et al.

Perilesional treatment of metastatic melanoma with interferon-beta. Clin Exp

Kakizaki A et al. Immunomodulatory effect of peritumoral administration of interferon-beta on melanoma through tumor-associated macrophages.

the et al. Phase I study of nivolumab combined with IFN-b for patients with advanced melanoma. Oncotarget 2017; in press.

Geant support

This study is partially funded by Ono Pharmaceutical Co., Ltd. and Bristol-Myers Squibb. 